Supplementary Materials for

\title{
Engineered bacterial production of volatile methyl salicylate
} Tiffany Chien ${ }^{1}$, Drew R. Jones ${ }^{4}$ and Tal Danino ${ }^{1,2,3} \dagger$

1Department of Biomedical Engineering, Columbia University, New York, NY 10027, USA. 2Data Science Institute, Columbia University, New York, NY 10027, USA. ${ }^{3}$ Herbert Irving Comprehensive Cancer Center, Columbia University, New York, NY 10027, USA. ${ }^{4}$ NYU School of Medicine, Metabolomics Core Resource Laboratory at NYU Langone Health, 550 First Avenue, New York, NY 10016, USA.

†Corresponding author. Email: td2506@columbia.edu

\section{This PDF file includes :}

Fig. S1-S4 


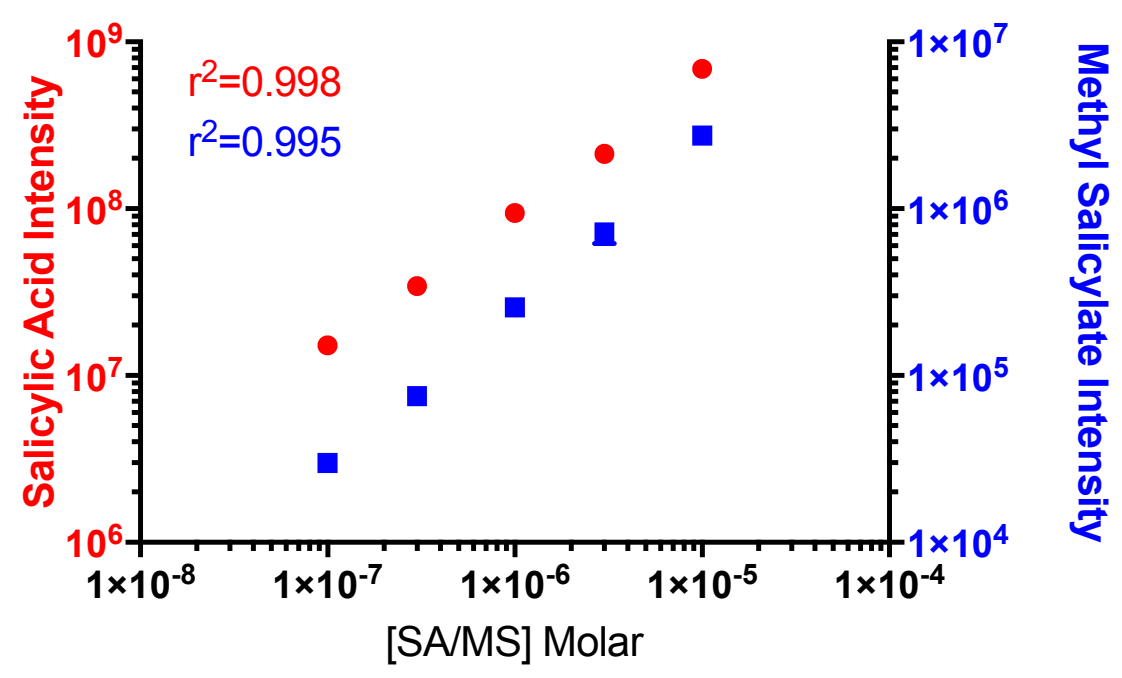

Supplementary Figure 1. Targeted LC-MS/MS Quantification of Salicylic Acid (SA) and Methyl Salicylate (MS). Calibration curves for SA and MS using authentic standards. Certified reference materials (Pharmaceutical Secondary Standards) were used to characterize both SA and MS by direct infusion prior to LC-MS/MS detection. The lowest calibrant was $100 \mathrm{nM}$ for each standard (5 femtomoles on column), with each calibrant run in technical duplicate. Error bars may appear smaller than symbols. 


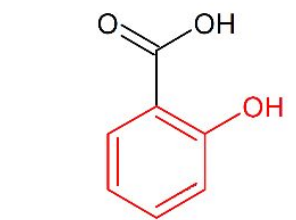

$137.0233 \rightarrow 93.0335$

$[\mathrm{M}-\mathrm{H}]^{-}$

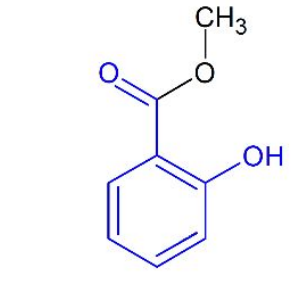

$153.0546 \rightarrow 121.0284$

$[\mathrm{M}+\mathrm{H}]^{+}$

Supplementary Figure 2. Identification of each compound in the experimental samples was based on accurate mass ( $3 \mathrm{ppm}$ ) and by MS/MS spectral match to the authentic standard. Quantification was based on the PRM (e.g., $137.0233 \rightarrow 93.0335 \mathrm{~m} / \mathrm{z}$ for salicylate) for each compound with a mass tolerance of $3 \mathrm{ppm}$ for the product ion. Colored segments of the chemical structure represent the atoms present in the MS/MS fragment detected in the PRM for each compound. Neutral chemical structures are shown for simplicity. 

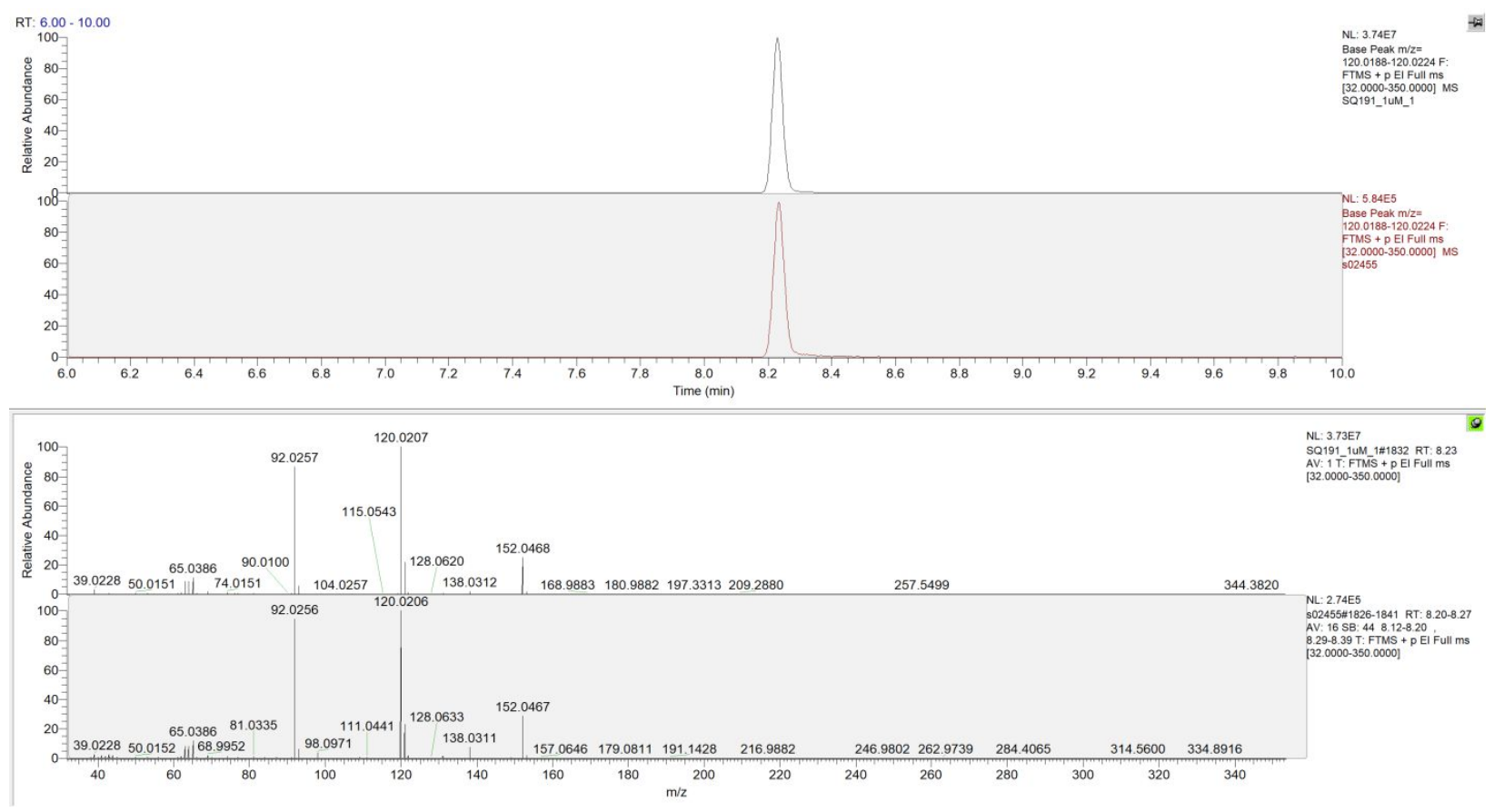

Supplementary Figure 3. Confirmation of Methyl Salicylate by GC-MS. Top chromatogram Extracted ion chromatogram showing the characteristic retention time for methyl salicylate in an authentic standard. Bottom chromatogram - detected peak in pTC035 media supernatant. Top spectrum - MS1 spectrum of the authentic standard peak, showing the intact ion at $152 \mathrm{~m} / \mathrm{z}$ and the characteristic fragment used for quantification at $120 \mathrm{~m} / \mathrm{z}$. Bottom spectrum - MS1 spectrum of the pTC035 peak, background subtraction has been used to highlight the methyl salicylate fragments matching the standard. 


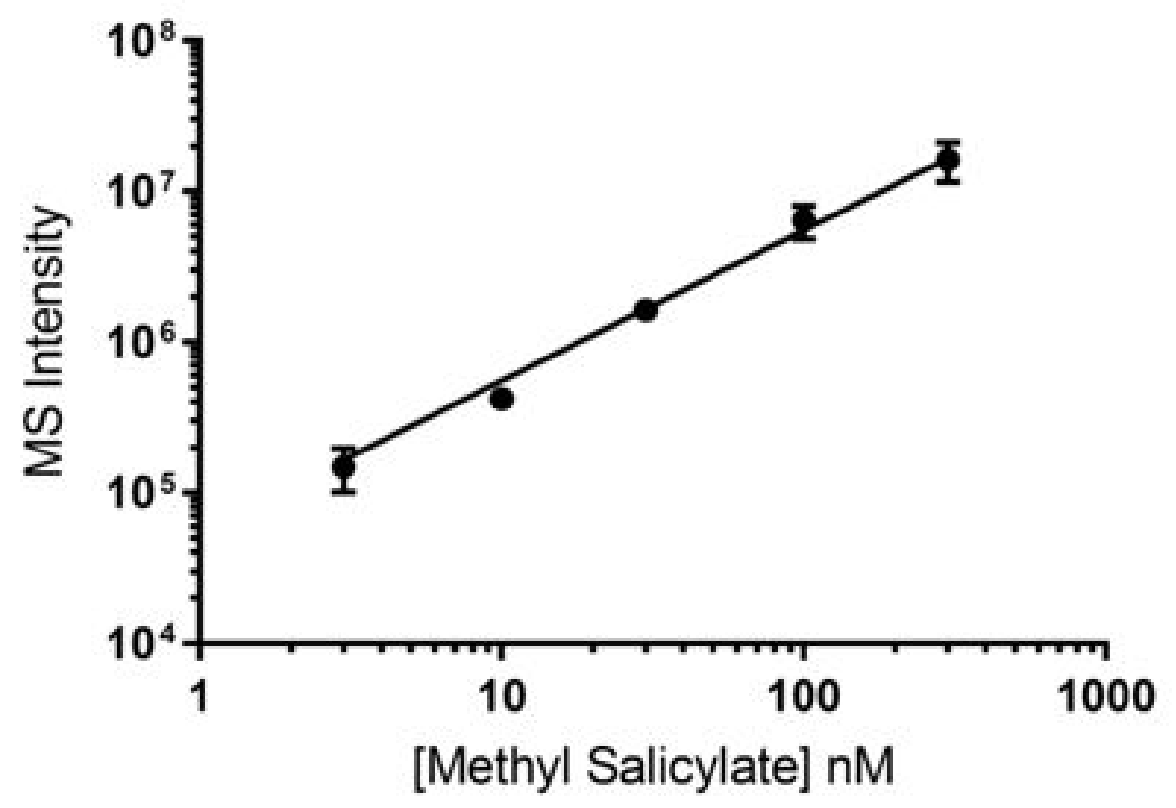

Supplementary Figure 4. Methyl Salicylate GC-MS standard curve. A linear regression was used to fit the standard curve, with a fixed origin through 0,0 . The resulting slope was $55273 \mathrm{MS}$ Intensity/nM Methyl Salicylate. 